\title{
Estratégias adaptativas de populações de Stylosanthes scabra provenientes de três regiões ecogeográficas de Pernambuco
}

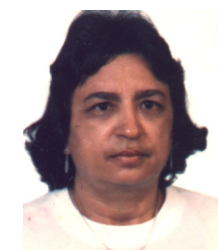

\author{
Rosilda de M. Queiroz', Gothardo Marcon², Clodoaldo J. da Anunciação Filho³ \\ Valderez P. Matos ${ }^{4} \&$ Roberta de A. Cisneiros ${ }^{5}$
}

\author{
1 UFRPE. Rua D. Manoel de Medeiros, s/n, CEP 52171-030, Recife, PE. Fone: (81) 3445-0596, Fax: (81) 445-2093. \\ Email: rosildaq@yahoo.com.br (Foto) \\ 2 UFRPE. Fone: (81) 3271-3703. E-mail: prpqp@girnpde.ufrpe.br \\ 3 UFRPE. Fone: (81) 3227-4785, Fax: (81) 3441-4798. Email: srmq@srmq.org \\ 4 UFRPE. Fone: (81) 3466-9348, Fax: (81) 3441-4798. Email: prpqp@girnpde.ufrpe.br \\ ${ }^{5}$ UFRPE. Fone: (81) 3439-5066, Fax: (81) 445-2093. Email: rosildaq@yahoo.com.br
}

Protocolo $020-20 / 02 / 2001$

Resumo: O presente trabalho teve como objetivo avaliar a variabilidade genética de 15 populações de Stylosanthes scabra (alfafa do nordeste) como resultado da estratégia adaptativa a diferentes ambientes das regiões da Mata, Agreste e do Sertão do Estado de Pernambuco. Estas populações, obtidas de coleta de amostras representativas de sementes, foram utilizadas em ensaio no Campo Experimental da Empresa Pernambucana de Pesquisa Agropecuária (IPA) o qual serviu de base para o estudo da distribuição fracionária de energia. A distribuição fracionária de energia para as partes da planta descrita em função da massa de matéria seca, classificou as populações como estrategistas $\mathrm{r}$ e $\mathrm{K}$, avaliadas durante o $1^{\circ}$ ciclo reprodutivo. Os resultados obtidos mostram existir grande variabilidade entre as populações para os caracteres avaliados. Brejos de altitudes selecionam populações de Stylosanthes scabra como estrategistas K. As populações do Sertão comportaram-se como estrategistas $r$, enquanto populações com maior precocidade reprodutiva, como as provenientes de Itamaracá, Serra Talhada e Santa Cruz da Baixa Verde, foram classificadas como estrategistas $r$, direcionando maior percentual de energia ao esforço reprodutivo. A grande variabilidade entre populações representa uma estratégia de adaptação aos diferentes ambientes, como processo de maximização genética na evolução da espécie.

Palavras-chave: alocação de energia, evolução, forrageira, leguminosa Stylosanthes

\section{Adaptive strategies in Stylosanthes scabra populations from three ecogeographic regions from Pernambuco - Brazil}

\begin{abstract}
This research aimed to evaluate the genetic variability of 15 Stylosanthes scabra populations (Alfafa do Nordeste) as a result of the adaptation strategy to different environments from the regions: Mata, Agreste and Sertão in the state of Pernambuco. These populations, obtained from representative samples of seeds, were planted in a field trial at Campo Experimental da Empresa Pernambucana de Pesquisa Agropecuária (IPA). This trial served as a base for the study of fractional energy distribution. The energy allocation to the plant parts, described as a dry matter weight function, classified the populations as $\mathrm{r}$ and $\mathrm{K}$ strategists, evaluated during the $1^{\text {st }}$ reproductive cycle. The obtained results show that there is a great interpopulational variability for all the evaluated characters. Based on the obtained data, Brejos de altitude target Stylosanthes scabra populations for K strategists. The populations from Sertão behaved as $r$ strategists. Populations with greater reproductive precocity, like that of Itamaracá, Serra Talhada and Santa Cruz da Baixa Verde, were classified as $r$ strategists, directing a higher percentage of energy relative to the reproductive effort. The great variability between populations represents an adaptation strategy to the different environments as a process of genetic maximization in the species evolution.
\end{abstract}

Key words: energy allocation, evolution, forage, leguminosae Stylosanthes 


\section{INTRODUÇÃO}

A distribuição de energia para os processos de crescimento, reprodução e sobrevivência dos organismos vivos, tem sido considerada decisiva para o entendimento da evolução, adaptação, seleção e história da vida desses organismos. A conexão com a história vital é observada através da relação dos tecidos reprodutivos com a fecundidade e dos tecidos vegetativos com a sobrevivência e/ou, futura fecundidade (Primack \& Antonovics, 1982).

Hamrick (1982) afirma que características de história de vida são aquelas diretamente envolvidas com o desenvolvimento da população, como sobrevivência, idade da primeira floração (ou reprodução), duração do tempo de fertilidade e alocação de energia para essas funções.

De acordo com Began et al. (1988) os conceitos de seleção r e K foi proposto por MacArthur \& Wilson em 1967 e desenvolvido por Pianka, em 1970. Esta teoria se fundamenta no fato de que os parâmetros $\mathrm{r}$ e K são determinados pela composição genética da população (Laroca, 1995).

MacArthur \& Wilson (1967) formularam a hipótese de que habitats sujeitos a alta taxa de mortalidade, independente da densidade, selecionarão espécies que alocam, proporcionalmente, maior esforço à reprodução (estrategistas do tipo r) enquanto habitats com taxas de mortalidade dependentes da densidade escolherão genótipos com maior capacidade competitiva, alocando, também, maior quantidade de energia às atividades de crescimento e manutenção (estrategistas do tipo K). Numa população em que atua a seleção tipo r, observa-se relação com uma história vital de habitat imprevisível e ciclo curto.

De acordo com MacArthur \& Wilson (1967) indivíduos de seleção tipo $r$ teriam as seguintes características: a) tamanho reduzido; b) amadurecimento precoce; c) maior assimilação reprodutiva nos indivíduos de menor tamanho; d) sobrevivência variável em função do ambiente.

Uma população de seleção tipo $\mathrm{K}$ vive em um habitat constante e previsivelmente estacionário no tempo. Essas espécies repartem suas energias em favor de sua manutenção e de melhor capacidade competitiva. Os estrategistas K competem pelo espaço ampliando seu porte, comportando as seguintes características: a) tamanho grande; b) reprodução retardada; c) menor assimilação reprodutiva; d) descendentes de maior tamanho e em menor número; e) investimento em uma sobrevivência maior, devido à intensa competição f) ciclo de vida perene.

Os conceitos de seleção $\mathrm{r}$ e K não são absolutos. Pode-se comparar espécies sendo, entretanto, observadas variações dentro da mesma espécie, em conseqüência de diferentes genótipos (Gadgil \& Solbrig, 1972).

Evenson (1983) afirmou que o método mais direto de determinação da alocação de energia dos tecidos de plantas se dá através da bomba calorimétrica. Entretanto, Harper \& Ogden (1970) introduziram um método de estudo de alocação de energia em plantas, o qual consiste em separar, de plantas individuais, suas diversas partes, de acordo com sua função, e expressar a alocação de energia como a proporção da biomassa seca total alocada a cada parte. Hickman (1975) \& Pitelka (1977) já consideravam não ser necessário o uso de calorimetria para a determinação dos padrões de alocação de energia em populações de plantas que acumulam primariamente carboidratos. Soares (1980) concluiu que, para as leguminosas herbáceas, a distribuição de biomassa seca fornece uma boa estimativa da distribuição de energia e, conseqüentemente, do esforço reprodutivo.

Jurik (1983) define esforço reprodutivo como sendo a energia total dedicada à reprodução, dividida pela energia total processada para a planta. De acordo com o autor, em termos gerais o esforço reprodutivo é definido como a proporção de recursos destinada à reprodução; entretanto, como uma estrutura pode freqüentemente ter duas ou mais funções, decisões sobre o que incluir no esforço reprodutivo são em geral arbitrárias.

Vários autores (Ogden, 1974; Gaines et al., 1974; Pitelka, 1977; Barriga, 1979; Primack \& Antonovics, 1982; Queiroz, 1998) mostraram que as espécies anuais e as populações precoces apresentam maior esforço reprodutivo que as espécies e/ou populações perenes e tardias, as quais alocam mais energia para a produção de biomassa vegetativa.

Além da importância da seleção r e K, no sentido evolutivo e ecológico, ressalta-se também a implicação do ponto de vista prático do melhoramento; assim, se o objetivo é a seleção para produção de sementes, deve-se trabalhar com aquelas populações mais do tipo $\mathrm{r}$ que do K; contrariamente, se o objetivo é a produção de massa verde para pastagens, trabalhar-se-á, então, com aquelas populações genéticas de seleção tipo K, que alocam mais energia para a produção de biomassa vegetativa (Reis, 1984).

Tratando-se de um dos aspectos centrais da genética ecológica, o estudo da dinâmica populacional que envolve, entre outros, o estudo das estratégias adaptativas a diferentes habitats, este trabalho teve como objetivo se estudar as estratégias de alocação de energia (seleção r e k) de populações obtidos de quinze acessos de Stylosanthes scabra pertencentes ao Banco de Germoplasma de Leguminosas Forrageiras da Universidade Federal Rural de Pernambuco (UFRPE) visando contribuir para um conhecimento maior desta espécie forrageira, por meio da avaliação de populações provenientes das regiões da Mata, Agreste e do Sertão do Estado de Pernambuco.

\section{MATERIAL E MÉTODOS}

Para realização do trabalho, instalou-se um experimento em campo, com as plantas em vasos, onde permaneceram até a época de corte, sob condições naturais de ambiente ao ar livre, em área da Empresa Pernambucana de Pesquisa Agropecuária - IPA, localizada no município de Recife, a $08^{\circ} 04^{\prime} 00^{\prime \prime}$, latitude S e $34^{\circ} 55^{\prime} 00^{\prime \prime}$ de longitude $\mathrm{W}$, e altitude de $4 \mathrm{~m}$.

As sementes de Stylosanthes scabra, que constituíram os acessos, foram coletadas de 15 populações e em média de 15 plantas por população, distribuídas nas regiões ecogeográficas da Mata, do Agreste e do Sertão do Estado de Pernambuco. As sementes de cada população foram reunidas em "bulks", mantendo-se a proporcionalidade das plantas que as compunham. As populações de Recife, Itamaracá, Paulista e Gaibu, representaram a região da Mata; Angelim, Arcoverde, Tacaimbó, Sanharó, Garanhuns e São Caetano, retrataram o Agreste; e Serra Talhada, Triunfo, Ibimirim I, Ibimirim II e Santa Cruz da Baixa Verde, a região do Sertão. 
Para se proceder à semeadura, as sementes de todas as populações foram previamente escarificadas com lixa, tendo-se o cuidado de não lesar o eixo embrionário. A semeadura foi realizada em 20/07/96, em placas de Petri e, no terceiro dia, as plântulas foram colocadas em copinhos de plástico instalados em telado, até o transplante para vasos de polietileno com $30 \mathrm{~cm}$ de profundidade, capacidade de $10 \mathrm{~kg}$ e apresentando $50 \mathrm{~cm}$ de diâmetro, que ocorreu em 20/09/96. O substrato se compunha de uma mistura de terra preta, esterco de curral curtido e argila, na proporção de 4:2:1, respectivamente. O delineamento experimental foi inteiramente casualizado, com três repetições e duas plantas em cada vaso.

Dados geoclimáticos dos municípios onde foram coletadas as amostras populacionais, estão contidos na Tabela 1.

Tabela 1. Dados geoclimáticos dos locais de coleta de amostras das populações estudadas

\begin{tabular}{lccc}
\hline Populações & $\begin{array}{c}\text { Altitude } \\
(\mathrm{m})\end{array}$ & $\begin{array}{c}\text { Temperatura } \\
\text { Média Anual } \\
\left({ }^{\circ} \mathrm{C}\right)\end{array}$ & $\begin{array}{c}\text { Precipitação } \\
\text { Média Anual } \\
(\mathrm{mm})\end{array}$ \\
\hline Recife & 15 & 24,0 & 1651,3 \\
Itamaracá & 25 & 25,6 & 2306,3 \\
Paulista & 50 & 24,5 & 1651,3 \\
Gaibu & 3 & 25,3 & 2213,9 \\
Angelim & 620 & 24,0 & 640,1 \\
Arcoverde & 700 & 26,5 & 640,1 \\
Tacaimbó & 530 & 26,0 & 661,2 \\
Sanharó & 620 & 22,4 & 708,5 \\
Garanhuns & 820 & 20,7 & 782,4 \\
São Caetano & 540 & 22,7 & 478,8 \\
Serra Talhada & 450 & 24,6 & 693,9 \\
Triunfo & 900 & 22,0 & 1227,6 \\
Ibimirim I & 520 & 24,5 & 453,5 \\
Ibimirim II & 600 & 24,5 & 453,5 \\
Santa Cruz da & & & $1227,6 *$ \\
$\quad$ Baixa Verde & 800 & 22,5 & \\
\hline Fontes: Instituto Nacional de Meteorologia (INEMET) 1997 e Empresa Pernambucana de Pesquisa \\
Agropecuária (IPA) 1997 \\
* Pluviosidade da estação mais próxima (Triunfo, PE)
\end{tabular}

\section{Distribuição fracionária de energia}

A comparação da distribuição fracionária de energia entre as populações estudadas foi realizada adotando-se a metodologia proposta por Harper \& Ogden (1970) que consiste em separar as várias partes da planta, de acordo com a sua função, e expressar a alocação de energia em função da biomassa seca. Para acompanhar a história de vida das populações, foram determinados o início do florescimento e da produção de sementes, e o final de reprodução de cada população durante o primeiro ciclo de vida que, neste trabalho, coincide com a época do corte das plantas. A colheita das sementes era realizada semanalmente. Os componentes da parte aérea que se desprendiam das plantas, como folhas, caules, ramos e frutos, foram acondicionadas separadamente, até a ocasião do corte de cada parcela.

Feito o corte na região do coleto, a parte subterrânea foi cuidadosamente lavada, seca em estufa e acondicionada em saco de papel.

Quando todas as populações já haviam sido coletadas, as diferentes partes de cada planta foram colocadas para secar a $75^{\circ} \mathrm{C}$ em estufa com circulação forçada de ar durante $48 \mathrm{~h}$ obtendo-se, a seguir, a massa seca de cada parte, em grama, utilizando-se balança de precisão de $0,01 \mathrm{~g}$.

\section{Procedimento estatístico}

Todos os dados coletados foram submetidos à análise de variância, e quando o valor de $\mathrm{F}$ foi significativo, as médias foram comparadas pelo Teste de Tukey a $5 \%$ de probabilidade (Gomes, 1981).

\section{RESULTADOS E DISCUSSÃO}

\section{Distribuição fracionária de energia}

A análise estatística dos dados referentes à alocação de energia, evidencia diferenças altamente significativas entre as populações de Stylosanthes scabra Vog. Os resultados estão representados na Tabela 2 e ilustrados na Figura 1.

Tabela 2. Massa seca de raízes (MSR), de caule + ramos (MSCR), de folhas (MSF) e de brácteas + flores + frutos (MSBFF) de 15 populações de Stylosanthes scabra, provenientes das regiões da Mata, do Agreste e do Sertão do Estado de Pernambuco

\begin{tabular}{|c|c|c|c|c|c|c|c|c|c|c|}
\hline \multirow[b]{2}{*}{ Região } & \multirow[b]{2}{*}{ Populações } & \multicolumn{2}{|c|}{ Raiz } & \multicolumn{5}{|c|}{ Esforço Vegetativo Aéreo } & \multicolumn{2}{|c|}{ Esforço Reprodutivo } \\
\hline & & MSR $^{*}$ & $\%$ & $\mathrm{MSCR}^{*}$ & $\%$ & MSF $^{*}$ & $\%$ & $\begin{array}{c}\text { Total }^{* *} \\
(\%)\end{array}$ & MSBFF $^{*}$ & $\%$ \\
\hline \multirow{3}{*}{ Mata } & Itamaracá & $2,900 \mathrm{f}$ & 8,95 & $8,203 \mathrm{f}$ & 25,33 & $8,183 \mathrm{e}$ & 25,26 & 50,59 & $13,013 \mathrm{bc}$ & 40,46 \\
\hline & Paulista & $16,860 \mathrm{de}$ & 15,22 & 51,426 de & 46,43 & 26,970 cde & 24,35 & 70,78 & $15,510 \mathrm{bc}$ & 14,00 \\
\hline & Gaibu & $15,037 \mathrm{e}$ & 16,86 & 37,743 def & 42,32 & 24,243 cde & 27,18 & 69,50 & $12,157 \mathrm{c}$ & 13,63 \\
\hline \multirow{4}{*}{ Agreste } & Tacaimbó & 22,903 cde & 17,10 & 58,287 cde & 43,51 & 31,197 cde & 23,29 & 66,80 & $21,563 \mathrm{abc}$ & 16,10 \\
\hline & Sanharó & $35,490 \mathrm{ab}$ & 15,85 & $100,563 \mathrm{a}$ & 44,92 & $70,373 \mathrm{ab}$ & 31,44 & 76,36 & $17,420 \mathrm{abc}$ & 7,78 \\
\hline & Garanhuns & $40,803 \mathrm{a}$ & 17,39 & $108,099 \mathrm{a}$ & 46,07 & $75,190 \mathrm{a}$ & 32,04 & 78,11 & $10,563 \mathrm{c}$ & 4,50 \\
\hline & São Caetano & $27,157 \mathrm{c}$ & 16,29 & $88,983 \mathrm{abc}$ & 50,90 & $40,923 \mathrm{~cd}$ & 24,51 & 75,41 & $13,893 \mathrm{bc}$ & 8,32 \\
\hline \multirow[t]{2}{*}{ Sertão } & Serra Talhada & $2,583 \mathrm{f}$ & 5,64 & $10,337 \mathrm{f}$ & 22,57 & $10,050 \mathrm{e}$ & 21,95 & 44,52 & $22,820 \mathrm{abc}$ & 49,84 \\
\hline & Triunfo & $26,260 \mathrm{c}$ & 12,16 & $91,203 \mathrm{ab}$ & 42,25 & $81,483 \mathrm{a}$ & 37,74 & 79,99 & $16,930 \mathrm{abc}$ & 7,84 \\
\hline C.V. & & 13,80 & & 18,84 & & 22,22 & & & 29,45 & \\
\hline D.M.S. & & 8,22 & & 31,10 & & 25,34 & & & 15,83 & \\
\hline
\end{tabular}

\footnotetext{
* Na coluna, médias seguidas por letras distintas diferem, pelo teste de Tukey, a nível de $5 \%$ de probabilidade
}

** Percentagem total de alocação de energia para estruturas vegetativas aéreas 


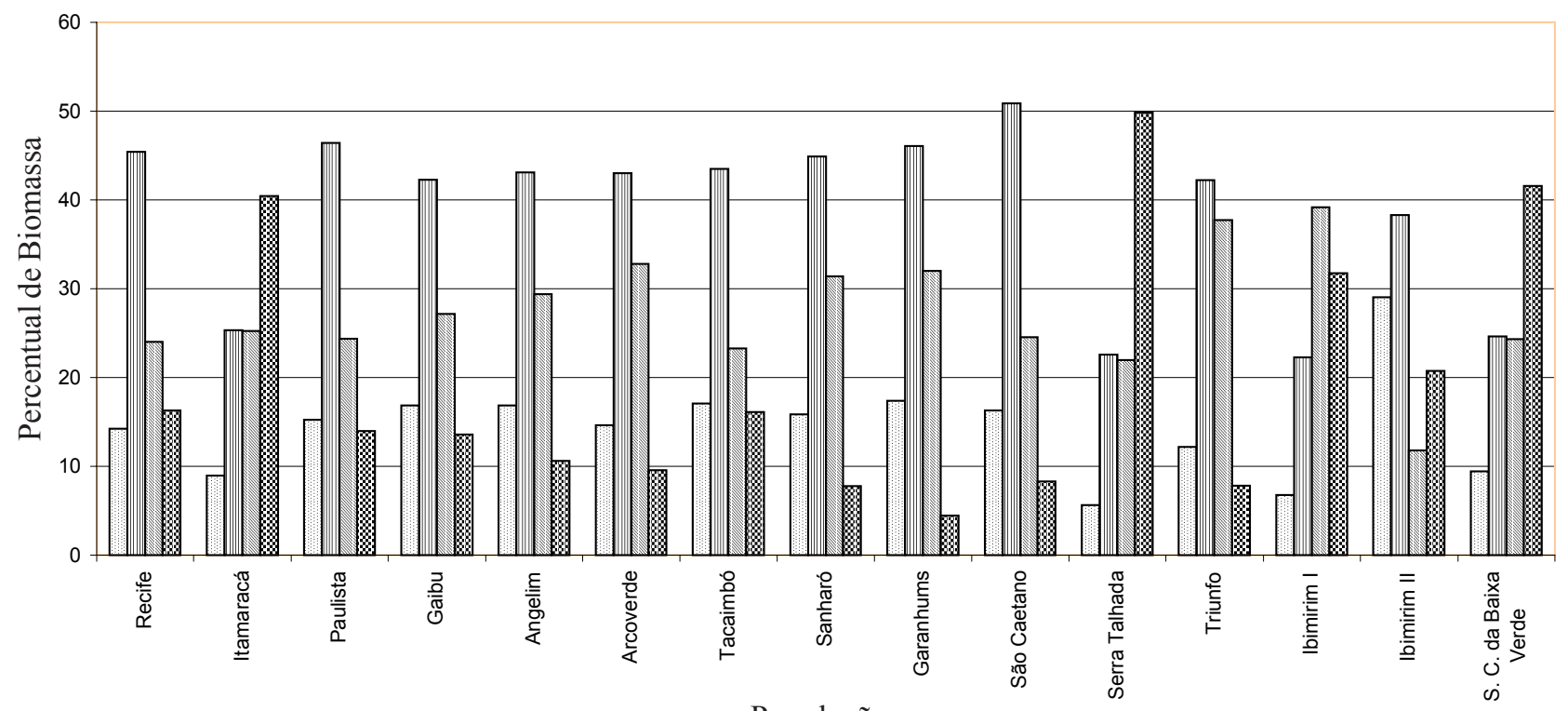

Populações

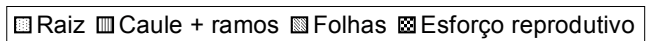

Figura 1. Distribuição fracionária de energia, expressa em percentagem de peso seco total de populações de Stylosanthes scabra (percentuais médios de 6 plantas por população)

Comparando-se os percentuais médios de distribuição de energia em cada população, nota-se que os maiores percentuais relativos à estrutura de reprodução foram observados para as populações de Itamaracá, Serra Talhada e Santa Cruz da Baixa Verde, com valores de 40,46, 49,84 e 41,56\%, respectivamente, enquanto os menores foram verificados para as populações Garanhuns (4,50\%) Sanharó (7,78\%) e Triunfo (7,84\%).

Verificam-se os maiores percentuais de energia distribuídos para caule + ramos nas populações de São Caetano, Paulista e Garanhuns, com os respectivos valores de 50,90, 46,43 e 46,07\%.

Segundo Gadgil \& Solbrig (1972) os conceitos de estrategista r e K não são absolutos e, sim, relativos. Pode-se comparar espécies sendo, entretanto, observadas variações dentro da mesma espécie, em conseqüência de diferentes genótipos. Conseqüentemente, a classificação de indivíduos é feita sempre em relação a outros indivíduos, em termos de biomassa alocada, à parte reprodutiva e vegetativa, em determinada condição ambiental.

Comparativamente, as populações Itamaracá, Serra Talhada e Santa Cruz da Baixa Verde, comportaram-se como estrategistas $r$ em relação às demais. Quando comparadas a estas, as populações Recife, Paulista, Gaibu, Angelim, Arcoverde, Tacaimbó, São Caetano, Ibimirim I e Ibimirim II, caracterizaram-se como estrategistas K mas, se comparar Ibimirim I e Ibimirim II com as demais, estas serão classificadas como estrategistas tipo $\mathrm{r}$, ao passo que as populações Garanhuns, Sanharó e Triunfo o serão como extremamente K. Sem dúvida, a evolução dessas plantas se deu como resultado da expressão de genes selecionadores dessas características, que conferem maior vantagem a genótipos que direcionam maior percentual de energia aos processos de crescimento e sobrevivência adequados ao ambiente.

Considerando-se as condições ecológicas dos brejos de altitudes como ambientes estáveis, observar-se-á a confirmação de que esses ambientes favorecem plantas estrategistas K. Vários autores (Gadgil \& Solbrig, 1972; Abrahanson \& Gadgil, 1973;
Gaines et al., 1974; Hickman, 1975; Newel \& Tramer, 1978; Abrahanson \& Gadgil, 1979; Jain \& Martins, 1979; Primack, 1979; Soares, 1980; Galloway, 1995) reportando-se à influência do habitat natural das espécies em sua estratégia adaptativa, evidenciaram que, à medida que o ambiente se torna mais evoluído na escala sucessional, há redução progressiva da fração de energia destinada à reprodução. As populações de ambientes instáveis, como as populações colonizadoras das margens de rodovias, por exemplo, alocam maior proporção de energia aos tecidos reprodutivos, quando comparadas com as populações de ambientes estáveis. Reis (1984) trabalhando com espécies do gênero Stylosanthes, concluiu que populações oriundas de ambientes estáveis destinavam maior quantidade de energia a estruturas vegetativas, dados confirmados nesta pesquisa.

Analisando-se a distribuição de energia direcionada às folhas, verificou-se que, dentre todas as populações estudadas, o menor percentual ocorreu na população Ibimirim II $(11,83 \%)$ e o maior para Ibimirim I $(39,18 \%)$. Ao se analisar as raízes, observa-se que o menor percentual entre as populações foi para Serra Talhada $(5,64 \%)$ e o maior para Ibimirim II $(29,07 \%)$. O comportamento das duas populações de Ibimirim demonstra grande variabilidade, que é resultado, certamente, de forte componente genético. Verifica-se, entre as populações, variação percentual média de 22,30 a $50,90 \%$ para os recursos alocados ao caule e ramos, enquanto para as folhas esta variação é de 11,83 a 39,18\%; para as raízes, de 5,64 a 29,07\% e, para o esforço reprodutivo, de 4,50 a $49,84 \%$. A maioria dos trabalhos realizados com o gênero Stylosanthes refere-se à sua ampla variabilidade genética e com considerável capacidade de adaptação a diferentes ambientes, podendo o gênero ser utilizado como pastagens nas mais variadas condições edafoclimáticas.

No presente estudo, pode-se observar que as populações Santa Cruz da Baixa Verde e Serra Talhada, de hábito semiprostrado, direcionaram maior percentual de energia à reprodução, contrariamente aos resultados de Soares (1980) 
que verificou que populações de hábito prostrado ou tendente a prostrado, alocaram maior quantidade de energia à produção de folhas e ramos. Também, populações com maior precocidade direcionaram maior percentual de energia a estruturas de reprodução quando comparadas às mais tardias (Tabelas 2 e 3). Soares (1980) trabalhando com Stylosanthes guianensis, concluiu não haver nenhuma analogia com a teoria preconizada de seleção $\mathrm{r}$ e $\mathrm{K}$, entre precocidade e esforço reprodutivo. No entanto, Reis (1984) trabalhando com oito espécies do gênero Stylosanthes, encontrou correlação positiva entre esforço reprodutivo e espécies que apresentaram maturidade sexual precoce.

Tabela 3. Número médio de dias decorridos da semeadura (20/07/96) ao início do florescimento, da produção de sementes e ao corte das plantas das populações de Stylosanthes scabra das regiões da Mata, Agreste e Sertão de Pernambuco

\begin{tabular}{lccc}
\hline \multirow{2}{*}{ Populações } & Florescimento & $\begin{array}{c}\text { Produção de } \\
\text { Sementes }\end{array}$ & $\begin{array}{c}\text { Corte das } \\
\text { Plantas }\end{array}$ \\
\cline { 2 - 4 } & \multicolumn{3}{c}{$(\mathrm{d})$} \\
\hline Recife & 75,67 & 133,67 & 216 \\
Itamaracá & 67,00 & 94,33 & 160 \\
Paulista & 74,00 & 112,00 & 167 \\
Gaibu & 68,67 & 102,67 & 174 \\
Angelim & 87,67 & 119,33 & 167 \\
Arcoverde & 83,67 & 114,00 & 167 \\
Tacaimbó & 80,67 & 118,33 & 167 \\
Sanharó & 85,67 & 109,00 & 195 \\
Garanhuns & 95,33 & 141,33 & 216 \\
S. Caetano & 96,33 & 124,33 & 195 \\
Serra Talhada & 60,67 & 93,00 & 160 \\
Triunfo & 93,00 & 124,33 & 202 \\
Ibimirim I & 88,33 & 113,00 & 160 \\
Ibimirim II & 84,67 & 109,00 & 167 \\
S. Cruz da B. Verde & 60,00 & 93,67 & 160 \\
\hline
\end{tabular}

Os resultados deste trabalho mostram a existência de grande variabilidade na alocação de energia entre as populações. Provavelmente, isto é resultado dos processos evolutivos a que foram submetidas. $\mathrm{O}$ aumento da variação genética em componentes de "fitness" expressos em um novo ambiente, influencia decisivamente as chances de desenvolver adaptações para os diferentes ambientes (Kawecki, 1995). Uma das mais importantes descobertas da genética evolutiva foi o achado da grande variabilidade genética que existe nas características de variação morfológicas (Suzuki et al., 1989).

Além do aspecto evolutivo, não se pode deixar de ressaltar a importância da espécie Stylosanthes scabra, do ponto de vista econômico, como forrageira. Genótipos oriundos das populações Triunfo, Garanhuns, Sanharó e Arcoverde, são altamente promissores como produtores de biomassa, pois alocaram os maiores percentuais de energia ao esforço vegetativo aéreo. De acordo com os resultados, essas populações oferecem boa alternativa para um programa de melhoramento, em que o objetivo seja a produção de massa verde para pastagens.

Observa-se que as populações mais precoces no florescimento são as que direcionam maior percentual de recursos ao esforço reprodutivo. Itamaracá, Serra Talhada e
Santa Cruz da Baixa Verde, apresentaram os maiores percentuais de alocação de recursos ao esforço reprodutivo, com valores de 40,46, 49,84 e 41,56\%, respectivamente (Tabelas 3 e 2). As populações mais tardias e que permaneceram maior tempo no campo, foram as que alocaram menor percentual ao esforço reprodutivo e, conseqüentemente, maior percentagem de material forrageiro. A populações Garanhuns e Recife foram tardias para a produção de sementes. A população Recife, mesmo tendo florescido em média com 75,67 dias, só frutificou aos 133,67 dias, com um percentual relativo alocado para reprodução de $16,30 \%$, superior ao das populações Paulista e Gaibu. Garanhuns floresceu aos 95,33 dias, porém produziu sementes aos 141,33 dias, apresentando alto desempenho em produção forrageira. Reis (1984) estudando espécies do gênero Stylosanthes, encontrou resultados semelhantes e concluiu que as espécies mais tardias para a produção de sementes que permaneceram mais tempo no campo até a época do corte, foram as que alocaram menor percentual de energia ao esforço reprodutivo, sendo selecionadas como estrategistas do tipo K. Soares (1980) trabalhando com Stylosanthes humilis H.B.K., constatou que as populações de florescimento precoce apresentaram maior esforço reprodutivo quando comparadas com as populações de florescimento tardio, sendo aquelas classificadas como estrategistas do tipo r. Com exceção da população Triunfo, considerada de brejo de altitude, as populações do Sertão foram as que alocaram maior percentual relativo ao esforço reprodutivo, quando comparadas com as da Mata e Agreste, sendo todas classificadas, portanto, como estrategistas do tipo $\mathrm{r}$ em relação às demais. Em média, as populações do Agreste levaram 121 dias para a produção de sementes, enquanto para as populações do Sertão e da Mata esses índices foram de 106,6 e 110 dias, respectivamente. Possivelmente, as populações do Sertão foram selecionadas para o florescimento precoce, por ser esta uma região cujo período de chuvas é antecipado em média de dois a três meses, em relação às outras duas regiões (Marcon, 1988).

\section{CONCLUSÕES}

1. Os brejos de altitude, Triunfo e Garanhuns, são ambientes que selecionam populações estrategistas K de Stylosanthes scabra.

2. Excetuando-se a população Triunfo, as demais populações do Sertão pernambucano comportam-se como estrategistas $r$.

3. Populações da região da Mata e do Agreste pernambucano, com exceção de Itamaracá, comportam-se como estrategistas K.

4. A produção forrageira em $S$. scabra é amplamente influenciada por diferenças ecológicas.

5. É possível selecionar-se populações de $S$. scabra como alternativa de melhoramento e garantia de pastagens.

6. As populações mais importantes sob o ponto de vista de utilização como forragem em pastejo direto, são: Ibimirim I, Triunfo, Arcoverde e Garanhuns.

\section{LITERATURA CITADA}

Abrahanson, W.G.; Gadgil, M. Growth form and reproductive effort in Goldenrods (Solidago compositae). American Naturalist, Chicago, v.107, n.957, p.651-661, 1973. 
Abrahanson, W.G.; Gadgil, M. Patterns of resource allocation in wild flower populations of fields and wood. American Journal of Botany, Columbus, v.66, n.1, p.71-79, 1979.

Barriga, J.P. Autoecologia de Stylosanthes humilis H.B.K.: Avaliação da variabilidade morfológica e estudos da biologia da semente. Piracicaba: ESALQ/USP, 1979. 97p. Dissertação Mestrado

Began, M.; Harper, J.L.; Cowsrsend, G.R. Ecologia, indivíduos poblaciones y comunidades. Barcelona: Omega, 1988. 215p.

Evenson, W.E. Experimental studies of reproductive energy allocation in plants. In: Little, R.J.; Jones, C.E. (ed) Handbook of experimental pollination ecology. New York: Scientific \& Academic Editions, 1983. p.249-274. 416p.

Gadgil, M.; Solbrig, O.T. The concept of $r$ and K selection: Evidence from wild flowers and some theoretical considerations. American Naturalist, Chicago, v.106, n.947, p.14-31, 1972.

Gaines, M.S.; Vogt, K.J.; Hamrick, J.L.; Caldwell, J. Reproductive strategies and growth patterns in sunflowers (Helianthus). American Naturalist, Chicago, v.108, n.964, p.889-894, 1974.

Galloway, F.L. Response to natural environmental heterogeneity: Maternal effects and selection on life-history characters and plasticities in Mimullus guttatus. Evolution, College Park, v.49, n.6, p.1095-1107, 1995.

Gomes, P.G. Curso de estatística experimental, $9^{\mathrm{a}}$ ed. Piracicaba: USP - Escola Superior de Agricultura Luiz de Queiroz, 1981. 430p.

Hamrick, J.L. Plant population genetics and evolution. American Journal of Botany, Columbus, v.69, n.10, p.1685-1693, 1982.

Harper, J.L.; Ogden, J. The reproductive strategy of higher plants. The concept of strategy with special reference to Senecio vulgaris L. Journal of Ecology, Oxford, v.58, n.119, p.681-698, 1970.

Hickman, J.C. Environmental unpredictability and plastic energy allocation strategies in the annual Polygonum cascadense (Poligonaceae). Journal of Ecology, Oxford, v.63, n.2, p.689-701, 1975.

Instituto Nacional de Meteorologia - INEMET. Dados meteorológicos mensais do Nordeste. Recife, PE. 1997.

Empresa Pernambucana de Pesquisa Agropecuária - IPA. Dados pluviométricos mensais do Nordeste. Pernambuco - Série Pluviométrica 6. Recife, PE. 1997.

Jain, S.K.; Martins, P.S. Ecological genetics of the colonizing ability of rose clover (Trifolium hirtum All.). American Journal of Botany, Columbus, v.66, n.9, p.361-366, 1979.
Jurik, T.W. Reproductive effort and $\mathrm{CO}_{2}$ dynamics of wild strawberry populations. Ecology, New York, v.64, n.6, p.1329-1342, 1983.

Kawecki, J.T. Expression of genetic and environmental variation for life history characters on the usual and novel hosts in Callosobruchus maculatus (Coleoptera: Bruchidae) Heredity, Oxford, v.75, n.1, p.70-76, 1995.

Laroca, S. Ecologia: Princípios e métodos Petrópolis, Rio de Janeiro: Vozes. 1995. 197p.

MacArthur, R.H.; Wilson, E.O. The theory of island biogeography. Princeton, New Jersey: Princeton University Press, 1967.203p.

Marcon, G. Estrutura genética de populações de Stylosanthes humilis H.B.K. (Leguminosae) de três regiões ecogeográficas do Estado de Pernambuco. Piracicaba: ESALQ/USP 1988. 179p. Tese Doutorado

Newel, S.J.O.; Tramer, E.J. Reproductive strategies in herbaceous plant communities during succession. Ecology, Durham, v.59, n.2, p.228-234, 1978.

Ogden, J. The reproductive strategy of plants. II. The reproductive strategy of Tussilago farfara. Journal of Ecology, Oxford, v.62, n.136, p.291-324, 1974.

Pianka, E.R. On r- and K-selection. American Naturalist, Chicago, v.104, n.927, p.592-597, 1970.

Pitelka, L.F. Energy allocation in annual and perennial lupines (Lupinus: Leguminosae). Ecology. Durham, v.58, n.5, p.1055-1065, 1977.

Primack, R.B. Reproductive effort in annual and perennial species of Plantago (Plantaginaceae). American Naturalist, Chicago, v.114, n.900, p.51-62, 1979.

Primack, R.B.; Antonovics, J. Experimental ecological genetics in Plantago. VII Reproductive effort in populations of $P$. lanceolata L. Evolution, Kansas, v.36, n.4, p.742-752, 1982.

Queiroz, R.M. Avaliação de Estratégias adaptativas de Stylosanthes scabra (Leguminosae- Papilionoideae) Vog. em três regiões ecogeográficas de Pernambuco. Recife: UFRPE, 1998. 117p. Dissertação Mestrado

Reis, M.S. Autoecologia de diferentes espécies de Stylosanthes Sw.: Análise da alocação de energia e estudos da biologia da semente. Piracicaba: ESALQ/USP 1984. 170p. Tese Doutorado

Soares, A.R. Ecologia de Populações de Stylosanthes guianensis (Aubl) Sw. (Leguminosae - Papilionoideae). Piracicaba: ESALQ/USP 1980. 85p. Tese Doutorado

Suzuki, D.T.; Griffths, A.J.F.; Miller, J.H.; Lewontin, R.C. Introdução à genética. 4. ed. Rio de Janeiro: Guanabara Koogan, 1989.633p. 